\title{
INTERVENIR EN LAS PREOCUPACIONES SOCIALES. UNA REFLEXIÓN SOBRE LA CORPORATE SOCIAL RESPONSIBILITY EN EL TURISMO EN ITALIA*
}

\author{
Tullio Romita ${ }^{1}$ \\ Università della Calabria
}

\begin{abstract}
Resumen: En el mundo "opulento" en el que hoy vivimos, se estabilizan, en lugar de desaparecer, los miedos y las ansias, las incertidumbres de las personas relacionadas con el futuro y la seguridad propia, y de la familia y los hijos, porque perciben una pérdida de garantías y tutelas sociales (educación, salud, trabajo, etc.) conquistadas a lo largo del tiempo, y también se debilitan -en lugar de reforzarse- las posibilidades de intervención del Estado social, en contraste con el desarrollo salvaje de la economía impuesta por los actores privados y donde se multiplican los trabajadores globalizados y descontentos, obligados a operar en una situación de creciente flexibilidad, movilidad, riesgo e incertidumbre.

Para solucionar el problema es necesaria una profunda reflexión sobre los problemas y los valores relacionados con la forma de actuar humana. Hay que destacar como central la cuestión del respeto de los principios de la justicia y de los individuos, que el problema es de tipo ético, tanto a nivel preceptivo como descriptivo, lo que quiere decir"la totalidad de normas de conducta pública y privada seguidas por las personas".

La Unión Europea, a través de un largo y en parte tortuoso recorrido, ha identificado en la ética de la empresa una de las cuestiones prioritarias en las que intervenir, tratando de promoverla en los países de la Unión. Este es el objeto de análisis de este trabajo, con especial referencia a la Responsabilidad Social Corporativa en el sector del turismo en Italia.
\end{abstract}

Palabras clave: la ética empresarial, las preocupaciones sociales, el riesgo, la incertidumbre, la sostenibilidad financiera, el código ético.

Resumo: Intervir nas preocupacións sociais. Unha reflexión sobre a responsabilidade social corporativa no turismo en Italia

No mundo «opulento» en que hoxe vivimos estabilízanse, en lugar de desaparecer, os medos e as ansias, as incertezas das persoas en relación co futuro e a seguridade propia, da familia e dos fillos, porque se percibe unha perda de garantías e tutelas sociais nos ámbitos da educación, a saúde, o traballo etc. conquistadas ao longo do tempo; tamén porque se debilitan -en lugar de se reforzaren- as posibilidades de intervención do Estado social, en contraste co desenvolve-

\footnotetext{
* Traducción de Lourdes Santisteban Regules.

1 Tullio Romita, Departamento de Sociología y de Ciencias Políticas - Centro Ricerche e Studi sul Turismo, Università della Calabria, Via P. Bucci Cubo 0B, 87036 Rende (CS), Italia. Tel. +39.0984.492554 - Fax +39.0984.492598 - E-mail: tromita@unical.it.
} 
mento salvaxe da economía imposta polos actores privados en que se multiplican os traballadores globalizados e descontentos, obrigados a operar nunha situación de crecente flexibilidade, mobilidade, risco e incerteza.

Para solucionar o problema cómpre unha profunda reflexión sobre os problemas e os valores relacionados coa forma de actuar humana. Neste sentido, hai que salientar como central a cuestión do respecto aos principios da xustiza e aos individuos, un problema que é de tipo ético tanto do punto de vista preceptivo como descritivo, o que quere dicir que atinxe «a totalidade de normas de conduta pública e privada seguidas polas persoas».

A Unión Europea, a través dun longo e en parte tortuoso percorrido, identificou na ética da empresa unha das cuestións prioritarias en que intervir, e tenta promovela nos países que a constitúen. Este é o obxecto de análise deste traballo, con especial referencia á responsabilidade social corporativa no sector do turismo en Italia

Palabras clave: ética empresarial, preocupacións sociais, risco, incerteza, sustentabilidade financeira, código ético.

\begin{abstract}
Intervening in social concerns. A reflection on Corporate Social Responsibility in tourism in Italy.

The fears, anxieties and uncertainties that people have in relation to the future and the safety of themselves and their family and children persist rather than disappear in the "affluent" world in which we live today, because they perceive a loss of the guarantees and social protection (education, health, work etc) conquered over time. In addition, the possibilities for state intervention, instead of becoming stronger, are becoming weaker, in contrast with the wild development of the economy imposed by private operators, where globalised and unhappy workers are multiplying, forced to operate in a situation of growing flexibility, mobility, risk and uncertainty.

A profound reflection on the problems and values related with human actions is required to solve the problem. It is important to highlight the core question of respect for the principles of justice and individuals, and that the problem is an ethical problem, both on a prescriptive and descriptive level, which means "the total sum of standards of public and private conduct which people follow".

The European Union, over a long and sometimes tortuous journey, has identified business ethics as one of the priority issues in which to intervene and promote in Union countries. This is the subject matter to be analysed in this work, with special reference to Corporate Social Responsibility in the tourism sector in Italy
\end{abstract}

Key words: business ethics, social concerns, risks, uncertainty, financial sustainability, the ethical code.

\title{
1. LAS PREMISAS.
}

La crisis económica actual ha provocado el pánico en millones de ciudadanos y ha dejado en la calle a miles de empresas de grandes y pequeñas dimensiones. La crisis ha afectado a los países más ricos e industrializados del mundo y ha obligado a los gobiernos a intervenir directamente y con recursos propios para tratar de limitar los efectos negativos, a la espera de encontrar una solución "política" que permita una recuperación de la economía.

La mayor parte de los analistas coinciden a la hora de considerar que, la causa principal reside en que durante muchos años, se ha permitido la práctica de una política económica que ha generado imperios financieros globales construidos sobre relucientes 
bases de cristal, capaces de reflejar una luz que ha deslumbrado a millones de personas, que las ha empujado a creer que sería posible alcanzar sus sueños sin demasiada dificultad, que el problema de la satisfacción de las necesidades primarias ya era un recuerdo lejano, que se podría ir más allá de lo superfluo (que arrogantemente invade nuestras casas y todos los demás ambientes públicos y privados en los que vivimos), que "quizás" se podría conquistar la inmortalidad.

Desde hace meses están llegando señales desde diferentes partes que indican que no ha sido así. Las bases de cristal no han soportado el peso de la enorme cantidad de individuos que, atraídos por su luz, habían terminado siendo el cuerpo de tales imperios. Las formas originarias se han hecho añicos, irreconocibles y sin brillo. Ahora, millones de personas están desorientadas y preocupadas, sin la luz que las había guiado, las había estimulado sobre las decisiones a tomar, las había encaminado hacia lo que parecía un futuro alentador y próspero.

Hace meses que los medios de comunicación nos recuerdan con continuidad estresante, que ha sucedido lo que nunca habríamos pensado que podría suceder. Crisis, recesión, paro, ayudas a las familias, apoyo a las clases sociales más pobres, aumento de la delincuencia común, quiebras, etc. Términos que han llevado a las personas de todo el mundo "rico" a un estado de frustración general que junto con estados de depresión, nos induce incluso a odiar los numerosos objetos inútiles que nos rodean, a menudo utilizando préstamos al consumo, que durante años nos han concedido fácilmente sin tener en cuenta nuestra capacidad de gasto real.

Los expertos y analistas económicos nos informan de que uno de los principales problemas para poder superar la grave fase de crisis en la que nos encontramos, será superar la cuestión de la pérdida de confianza de las personas en los mercados y en los gobernantes. $\mathrm{Y}$ en efecto, la cuestión no es nada fácil de solucionar, ¿cómo se pretende que se tenga confianza en el mercado y/o en el Estado a uno de los tantos americanos que en los últimos años han adquirido una vivienda y que, como consecuencia de mecanismos financieros falsos y /o del desplome del mercado inmobiliario, hoy no tienen una casa propia porque no han sido capaces de devolver el préstamo concedido por los bancos para su compra, o que poseen una vivienda que vale la mitad del precio pagado hace sólo un par de años?; ¿a uno de los tantos trabajadores del sector automovilístico que en alrededor de un mes ha perdido su trabajo o se encuentra ante un expediente de regulación de empleo?; ¿a las miles de personas pertenecientes a la llamada clase media que ven disminuir su propio poder adquisitivo, arriesgándose a deslizarse "peligrosamente" hacia un estatus más bajo?; ¿a las personas intoxicadas por lo que, fiándose, compran y consumen?; etc.

Consideramos bastante obvio que no es nada fácil convencer a estas personas de que el mejor modo para reaccionar ante la crisis económica mundial no es asustarse y agarrotarse, sino intentar llevar una vida normal, si acaso reaccionando a la mala suerte asumiendo comportamientos activos orientados a restablecer las condiciones originarias. La situación negativa ha sido la consecuencia de políticas gubernamentales complacientes con el libre mercado, de vías de crecimiento más basadas en la economía virtual que 
en la real, de modelos de desarrollo social que han privilegiado casi exclusivamente la creación del beneficio y de la riqueza económica y puesto en segundo plano modelos de desarrollo sostenible. Por lo tanto, la actual desconfianza respecto a los mercados y a los gobiernos está más que justificada.

Para solucionar el problema de la falta de confianza es necesario llevar a cabo una profunda reflexión sobre los problemas y los valores relacionados con la forma de actuar humana. Hay que destacar la necesidad de recuperar la centralidad del respeto de los principios de la justicia y de los individuos, tratando a éstos últimos, como ha escrito Kant “... siempre como fines y nunca como medios”. En conclusión, la crisis económica mundial pone en primer plano el hecho de que el problema es ante todo de tipo ético, tanto a nivel preceptivo como a nivel descriptivo lo que quiere decir "el conjunto de las normas de conducta pública y privada seguidas por las personas".

En este trabajo estamos haciendo referencia de forma específica al problema de la ética de empresa que de hecho, aunque no siempre se define explícita y comúnmente como tal, desde hace tiempo estaba despertando el interés de todo el mundo.

La continua e intensa expansión de la globalización de la economía, las apremiantes aceleraciones y las continuas revisiones (incluso durante su realización) de los comportamientos individuales y colectivos necesarios para favorecer los procesos de globalización, han establecido como único criterio positivo de evaluación de las alternativas y de los comportamientos, la competencia global: la "hipercompetencia". Sin embargo, de forma paralela a esta manera de actuar, en nuestras mentes, las de quienes estamos obligados a correr cada vez más rápidamente, se ha desarrollado la conciencia de que toda la sociedad corre grandes riesgos actuando de esta manera y también de la inutilidad de los resultados producidos para amplios sectores de la población.

Un mundo en pedazos, en el cual, paradójicamente, con el aumento de la globalización de los mercados y de las posibilidades de comunicación se acentúan, en lugar de atenuarse, las diferencias y las divisiones étnicas y culturales (Geertz, 1995); se consolidan, en lugar de desaparecer, las relaciones con los territorios y los lugares en los cuales se ha nacido y crecido y en los que se vive, a través de unas ganas enormes de formar parte de una comunidad que nos devuelve la sensación de vivir en ambientes y en espacios protegidos porque los conocemos bien, más reducidos, en los que instaurar relaciones personales directas, y en los que es posible encontrar una conexión con nuestras raíces (Bauman, 2001); se intensifican, en el lugar de limitarse o anularse, los conflictos relacionados con la gestión de los recursos comunes, mientras que el agua, el aire, los recursos culturales, e incluso el poco petróleo que todavía queda, deberían considerarse bienes accesibles y utilizables por todos, la base sobre la que construir un desarrollo económico y social equitativo, pacífico y duradero a lo largo del tiempo (Shiva, 2003); se estabilizan, en el lugar de desaparecer, los miedos y las ansias, las incertidumbres de las personas relacionadas con el futuro y la seguridad propia y de la familia y los hijos, ya que perciben una pérdida de garantías y tutelas sociales (educación, sanidad, trabajo, etc.) conquistadas a lo largo del tiempo (Beck, 2000); se debilitan, en el lugar de reforzarse, las posibilidades de intervención del Estado social en contraste con 
el desarrollo salvaje de la economía impuesta por los actores privados, principalmente las corporation (Brecher, Costello, 2001); se multiplican, en el lugar de disminuir, los trabajadores globalizados y descontentos, que son útiles e indispensables para el desarrollo de la economía global y de las multinacionales, y cada vez más obligados a operar en una situación de creciente flexibilidad, movilidad, riesgo e incertidumbres (Sassen, 2002; Sennet, 2001).

La globalización entra prepotentemente en la vida cotidiana y condiciona el destino de millones de personas, y mientras se invocan nuevas estrategias de vida, las personas (los jugadores) que habitan en este universo tambaleante viven en una condición de evidente incertidumbre (Bauman, 1999). La gran crisis actual obliga a afrontar las importantes injusticias, los cuantiosos daños al medio ambiente y las amplias contradicciones, generadas por el modelo de desarrollo contemporáneo.

Por tanto, el problema de la cuestión ética es una de las más importantes para llegar a un modelo de desarrollo más "sostenible", y sobre ésto, las instituciones pueden y deben jugar un papel fundamental.

Seguramente han sido de interés algunas actuaciones de la Unión Europea que, en el intento de encontrar soluciones a las cuestiones planteadas por las continuas y nuevas preocupaciones sociales surgidas en los últimos años, y a través de un largo y en parte tortuoso recorrido, ha identificado en la ética de empresa uno de los problemas prioritarios en los que intervenir. Un acto significativo en tal sentido, ha sido la promoción de la Responsabilidad Social Empresarial en los países de la Unión, y que constituye el objeto de análisis del presente trabajo.

\section{ORÍGENES Y DESARROLLO.}

El proceso mediante el cual se ha llegado a determinar la necesidad de promover la adopción, en la Unión Europea, de prácticas dirigidas a orientar a las empresas hacia la responsabilidad social, ha sido largo y lleno de obstáculos, y cuyo acto final de referencia ha sido el Libro Verde elaborado por la Comisión Europea en 2001.

Se puede decir que el recorrido ha iniciado cuando, razonando sobre las posibilidades de reforzar y potenciar las opciones para competir a nivel económico global, entre las acciones identificadas se ha asignado un papel relevante al desarrollo del sentido de responsabilidad colectivo de los diferentes sujetos públicos y privados. A partir de esta decisión, recogida en el conocido Libro Blanco elaborado por la Comisión Delors en 1993, los siguientes Consejos Europeos (Lisboa 2000 y Goteborg 2001) añadieron otros objetivos a perseguir para incrementar la capacidad de competencia económica en los países UE. Las palabras clave incluidas en la nueva ordenación del desarrollo económico futuro fueron: cohesión social, orientación hacia la sostenibilidad, calidad ocupacional, respeto del medio ambiente, innovación tecnológica.

El Libro Verde sintetiza e interpreta las instancias enunciadas introduciendo, como hemos señalado anteriormente, el principio de la responsabilidad social empresarial como criterio guía de las empresas en su forma de operar a nivel interno y de relacionar- 
se con el exterior, y a las que atenerse, también con la finalidad de obtener ventajas competitivas en el mercado global.

En efecto, el trabajo del organismo europeo ha producido consensos y efectos, no sólo por ser el fruto de años de análisis y estudios exhaustivos y convincentes, o porque ofrecía herramientas conceptuales útiles para afrontar de forma más eficaz la competencia y el crecimiento futuro de la empresa, sino también porque -llegado este momento de fuerte estrés de la sociedad a nivel mundial, que ha afectado indistintamente a operadores económicos, trabajadores, gobiernos nacionales y consumidores, como consecuencia de las continuas aceleraciones y reorganizaciones impuestas por los procesos de globalización- proponía una lectura convincente de la sociedad, y una posible solución para superar, al menos parcialmente, los problemas que caracterizaban el desarrollo económico y social a nivel mundial.

En efecto, la nueva orientación propuesta sobre el desarrollo económico, ha obtenido la adhesión de los gobiernos nacionales, y no solo a nivel de UE, sugiriendo, en algunos casos, intervenciones destinadas a promover la difusión y la aplicación del principio de la responsabilidad social empresarial voluntariamente, y en otros casos, a través de intervenciones de tipo legislativo. Por ejemplo: Francia e Inglaterra han desarrollado dispositivos legislativos que, modificando las reglas económicas y el derecho societario y comercial, obligan a las empresas que cotizan en Bolsa a realizar informes anuales sobre el balance social y medio ambiental; Italia ha puesto en marcha proyectos con el objetivo de promocionar la cultura de la RSE a través de la adhesión a los sistemas de certificado medio ambiental y a la aplicación de relaciones de RSE, a la co-financiación por parte de las empresas de proyectos relacionados con la cuestión social (Social Commitment); las Naciones Unidas han emanado un documento en el cual han recogido las obligaciones de las empresas multinacionales en materia de tutela y respeto de los derechos humanos fundamentales: igualdad de oportunidades, seguridad de las personas y los trabajadores, protección medio ambiental, etc.

Hay destacar que los compromisos asumidos y las acciones realizadas corresponden a una situación de creciente sensibilidad hacia la problemática RSE pero, también, a un conjunto de intervenciones concretas de difícil cuantificación y valoración de los efectos producidos respecto al cambio de los procesos de la empresa. Sin embargo, parece que hay un acuerdo tácito a la hora de considerar que las consecuencias más significativas están relacionadas con la mayor atención hacia el impacto medio ambiental de las actividades de empresa, los derechos de los trabajadores y la propia reglamentación interna de la práctica de la RSE.

En conclusión, por medio de la RSE se están abriendo lentamente, aunque incesantemente, nuevas vías hacia una nueva forma de gestionar la empresa, en las que el beneficio sigue siendo, inevitablemente, el objetivo prioritario, pero que a través del respeto del principio de la responsabilidad social, puede ser perseguido, como señalan algunos, en mayor medida y de forma más rápida, controlando las externaciones negativas de la actividad empresarial que genera el beneficio y respetando los derechos de quien contribuye directamente a la producción del beneficio. 


\section{DEFINICIONES Y PRINCIPIOS}

El Libro Verde de la Comisión Europea define la Responsabilidad Social de la Empresa como: "la inclusión voluntaria de las preocupaciones sociales y ecológicas de las empresas en sus operaciones comerciales y en sus relaciones con las partes interesadas".

Pero, como sabemos, pasando de la definición de conceptos y principios a las acciones y a las intervenciones que se quieren aplicar concretamente, se termina introduciendo muy a menudo e involuntariamente, elementos operativos que, en ocasiones, aunque sólo sea mínimamente, modifican su significado originario. Tal eventualidad, como se puede intuir, es todavía más probable que se verifique cuando la definición debe aplicarse en diferentes contextos culturales y/o periodos. Lo que ocurre es que como consecuencia de acciones y pensamientos, se introducen innovaciones que terminan modificando, ampliando, dilatando, enriqueciendo la definición originaria. Esto es lo que destaca Gallino (2007), quien siguiendo este razonamiento y en la base a la documentación nacional e internacional relacionada con la RSE incluida en el Libro Verde 2001 de la Comisión Europea, sostiene que la noción de RSE italiana tendría que modificarse. Según el estudioso italiano, la definición de RSE que parece emerger progresivamente a nivel internacional podría formularse así: "una empresa puede definirse responsable bajo el perfil económico, social y medio ambiental cuando y en la medida en que elija incluir en el cuadro decisional que dirige, sus estrategias societarias y las prácticas de gestión de todas las unidades productivas controladas por la misma bajo cualquier concepto, las normas, las cláusulas, las sugerencias, las prohibiciones, las recomendaciones, las obligaciones, a menudo de naturaleza moral y no jurídica, recogidas en los acuerdos y en las convenciones internacionales citadas en tales documentos, o sea las consecuencias de su violación o elusión, tanto si están recogidos formalmente como si no por la legislación vigente en los países en los cuales las empresas tienen su sede legal o en los que operan a través de empresas subsidiarias, empresas controladas (sea cual sea la base de este control), o cadenas de suministro" 2 .

En cualquier caso, los principios a los que hace referencia la definición son fundamentalmente tres:

1) las empresas deben decidir voluntariamente su adhesión a la lógica de la RSE a través de acciones visibles y concretas. La lógica en la que se basa tal principio es bastante evidente: el valor de comportamientos dirigidos a tutelar ansias e inquietudes sociales es mayor cuando es fruto de elecciones y decisiones tomadas voluntariamente y no por imposición de la ley;

2) las empresas tienen la obligación moral de emprender acciones que no perjudiquen los derechos de todas las partes interesadas por/en la propia actividad, y que pueden ser sujetos tanto internos como externos a la misma. Tales sujetos

2 Cfr. El informe de Luciano Gallino, Presidente de la Fundación I-CSR (www.i-csr.org), el Multi-stakeholder Forum sobre la Responsabilidad social de las empresas, promovido por el Ministerio para la Solidaridad Social en Roma el 19 de Diciembre de 2007. 
se denominan stakeholders (portadores de intereses), y pueden definirse como personas que, bajo diversos conceptos, individualmente o en grupo, y en todo caso interesados, contribuyen con sus comportamientos a definir la performance positiva de la empresa ${ }^{3}$...

3) la adopción de prácticas de gestión empresarial coherentes con el criterio de la RSE tienen el valor de herramientas estratégicas de gestión empresarial. De hecho, actuar de forma responsable respecto a los propios trabajadores, el medio ambiente, los territorios y las comunidades locales implicadas, las personas que se beneficiarán de lo que se produce, todos los sujetos con los que se interacciona, tiene repercusiones positivas tanto dentro de la propia empresa, como respecto a la propia imagen y reputación externas.

\section{DESTINATARIOS}

Los destinatarios de los procesos y de las acciones de la RSE son básicamente dos: quienes participan activamente y desde dentro en la vida y en el éxito de la empresa y quienes, bajo conceptos diferentes, contribuyen a su afirmación y desarrollo, a pesar de ser sujetos externos a la empresa. Por otro lado, no podía ser de otra manera porque, también como consecuencia de lo dicho hasta ahora, y de lo indicado en otras ocasiones, es fácil deducir que: "la atención de los operadores, de la opinión pública y de todos los portadores de intereses se concentra en la integridad de las empresas y en las actitudes adoptadas por éstas, no sólo en relación con los accionistas sino también con la sociedad en su conjunto. Para entender correctamente este reto, es necesario invertir más en el capital humano, en el medio ambiente y en las relaciones con las partes interesadas".

El trabajador, el propio empleado es el sujeto interno, al cual la empresa que aplica los principios de la RSE dedicará una especial atención. El empleado debe ser considerado un elemento activo, tanto respecto al crecimiento, al desarrollo y a la capacidad de competir de la empresa, como respecto a las posibilidades de crecimiento profesional y de afirmación social. Esto, según la RSE, sólo es posible si la empresa invierte en sus propios trabajadores, favoreciendo la puesta al día y la cualificación profesional, la participación y la implicación en los procesos productivos, la tutela de la salud y de la seguridad en el trabajo. Para la empresa que adopta tal filosofía, las repercusiones serán positivas respecto a las posibilidades de éxito a corto y largo plazo. En conclusión, desarrollar una relación que no sea "sólo de trabajo", sino también de colaboración y participación, significa para la empresa y para el trabajador, haber entendido el valor del "capital humano" para mejorar los resultados de la empresa.

3 Según el Libro Verde, stakeholder es: "Un individuo, una comunidad o una organización que influye en las operaciones de una empresa o sufre sus repercusiones. Los sujetos interesados pueden ser internos (por ej.: trabajadores dependientes) o externos (por ej.: consumidores, proveedores, accionistas, financiadores, la comunidad local)." Cfr. Comisión de la Comunidad Europea, Libro Verde. Fomentar un marco europeo para la responsabilidad social de las empresas, Bruselas, 2001. 
Comunidades locales, partners comerciales y clientes, son los sujetos externos a los cuales en la óptica RSE es necesario considerar de forma especial y nueva. Como señala el Libro Verde, "las empresas contribuyen al bienestar de las comunidades, sobre todo locales, proporcionando puestos de trabajo, salarios y prestaciones e ingresos fiscales. Inversamente, las empresas dependen de la buena salud, de la estabilidad y de la prosperidad de las comunidades que las acogen". Y de la colaboración entre empresas y comunidades locales puede surgir un mecanismo capaz de aumentar el capital social ${ }^{4}$ de la comunidad, factor que puede tener repercusiones positivas relevantes también en sectores de actividad económica diferentes de los prevalentes a nivel local (por ejemplo, mayores cuotas de capital social son un elemento estratégico importante para el sector turístico).

También es fundamental a nivel estratégico para el éxito de la empresa la relación que instaura con el usuario final, es decir, el cliente. Comportamientos socialmente responsables, orientados a satisfacer las expectativas y las necesidades de los usuarios, aumentan la credibilidad, la confianza y la reputación de la empresa.

La última cuestión de extrema relevancia para el éxito de la empresa respecto a las indicaciones de la RSE, es el respeto de los derechos humanos y de las preocupaciones medio ambientales a nivel mundial. La empresa que asume comportamientos responsables respecto al medio ambiente, no sólo reduce costes, sino que también genera una mayor disponibilidad y una mayor participación en todos los stakeholders implicados.

\section{PALABRAS CLAVE.}

Aplicar los consejos del Libro Verde y, por tanto activar procesos de responsabilidad social en la empresa, significa adoptar algunas herramientas que cuando se utilizan, hacen que tales procesos sean visibles para todos los stakeholders. Las palabras clave más difundidas en la RSE son: Balance Social, Balance Medio Ambiental, Balance de Sostenibilidad, Buenas Prácticas y Código Ético.

Los Balances son herramientas de gestión y de comunicación, cada una de las cuales se refiere a una dimensión de la RSE: lo social atañe a las relaciones entre la empresa y los portadores de intereses internos y externos de la misma; el medio ambiental se refiere a los impactos de tipo ecológico de las actividades empresariales; el de sostenibilidad sirve para hacer una valoración de las perspectivas de las generaciones futuras de poder disponer de las riquezas producidas y del medio ambiente en el que la empresa lleva a cabo su actividad.

4 Limitándonos a la definición del Libro Verde: "El patrimonio de actitudes y creencias compartidas por una determinada comunidad. El capital social constituye uno de los pre-requisitos de la cooperación y de la actividad organizada en las comunidades humanas, incluidas las actividades económicas. El capital social puede ser transformado, consumido o reconstituido, exactamente igual que el capital financiero." Cfr. Comisión de las Comunidades Europeas, Libro Verde. Fomentar un marco europeo para la responsabilidad social de las empresas, Bruselas, 2001. 
El Código Ético es la declaración de principios de las empresas, a la que hay que atenerse respecto al comportamiento a adoptar con todos los sujetos internos y externos a la empresa.

Las Buenas Prácticas (o más comúnmente, best practice) son acciones de RSE que, por la experiencia positiva obtenida, pueden y deben ser consideradas un punto de referencia para las demás empresas y para los stakeholders.

\section{RSE Y EMPRESA TURÍSTICA EN ITALIA}

El año siguiente a la publicación del Libro Verde, el Ministerio del Trabajo y de las Políticas Sociales propuso un programa para la promoción y la difusión de la cultura de la RSE entre las empresas italianas. Más adelante, en 2003, por medio del proyecto RSESocial Commitment, el gobierno italiano consideró necesario proponer unos parámetros útiles para favorecer la evaluación de la difusión de la RSE entre las empresas italianas.

En realidad, hoy en día todavía no existe un verdadero Observatorio que permita registrar información precisa y sistemática sobre la difusión y las modalidades de difusión de la RSE en las empresas italianas. Sin embargo, podemos afirmar tranquilamente que sobre el tema de la RSE en Italia objetivamente se ha obtenido un resultado: se ha asistido a un continuo florecer de páginas más o menos especializadas en internet y de asociaciones y entes que tienen como misión la promoción de la responsabilidad social de empresa. En cualquier caso, los estudios y las investigaciones realizadas nos permiten obtener información relevante sobre las últimas tendencias relativas a la RSE en Italia.

En general, podemos decir que la RSE todavía no ha entrado de forma difusa en las empresas italianas. En efecto, la impresión es que, incluso cuando se adoptan la lógica y la práctica de la RSE, las acciones parecen ser más actos obligados o, en todo caso necesarios para competir, y no la consecuencia de la toma de conciencia de la utilidad social efectiva de la RSE que, inevitablemente, conduce a cambiar la misión y la razón de ser de la empresa (como se propone) $)^{5}$.

En Italia, la adopción de la RSE todavía se considera algo que atañe a las grandes empresas, y a la industria manufacturera en particular. Pero el tejido productivo italiano es similar al europeo, y por tanto está caracterizado por la presencia de una extensa red de pequeñas y medianas empresas (de ahora en adelante "PMI").

La sensibilidad de las PMIs italianas hacia la RSE parece ser especialmente actual. Alrededor del 45\% de las mismas ha obtenido el certificado SA8000 (relativo al respeto de los derechos humanos y contra la explotación de los menores), uno de los porcentajes de difusión más elevados a nivel europeo ${ }^{6}$. Respecto a las PMIs que cotizan en la

5 El estudio publicado por el Observatory of European SMEs (European Commission, 2002), por ejemplo, señala que la mitad de las PMIs europeas realiza alguna intervención a favor de la comunidad externa, si bien tales compromisos son fundamentalmente ocasionales y no están relacionados con la estrategia empresarial. 6 Hay un estudio sobre las pequeñas empresas centrado en una de las áreas más importantes de la RSE, la de las relaciones con los trabajadores: Employment Relations in Micro and Small Enterprises in the EU Literature Review (European Foundation for the Improvement of Living and Working Conditions, 2001). 
Bolsa, las que han adoptado voluntariamente políticas de RSE son sólo el 15\%, muy por debajo de la media europea, que sin embargo alcanza el $60 \%$ en el caso de las empresas que aplican el índice Standard and Poor's MIB.

El hecho de que entre las PMIs italianas exista un gran interés hacia la RSE, depende de diferentes factores, en todo caso relacionados con el hecho que las mismas están, en la gran mayoría de los casos, radicadas en el territorio y en las comunidades locales en las que operan, y que, como otros ya han señalado, estos factores son: "el apego de muchas de ellas a valores -católicos, liberales y socialistas - que promueven un interés sobre las expectativas de los trabajadores y sus familias (Rebora, 1984); el valor social propio de los términos productivos, que se convierten en áreas de desarrollo de los conocimientos técnicos y comerciales, de la cultura emprendedora, de estructuras educativas y normativas, de servicios a los trabajadores y a sus familias (Belussi, Gottardi, Rullani, 2000); la prevalencia de las empresas familiares, a menudo caracterizadas por una gestión positiva de las relaciones con los colaboradores (Quinn, 1997)"”.

Este apego al territorio y a la comunidad local, al hecho de ser más fácilmente reconocibles, localizables y alcanzables que las grandes empresas, hace que las PMIs italianas presten más atención a las presiones de los stakeholders y en particular de los consumidores. ONGs, accionistas y consumidores son, según la European RSE Survey de 2007, los stakeholders europeos que más condicionan las decisiones de la empresa. Entre éstos, los consumidores son los sujetos que en los últimos años han tomado mayor conciencia de la posibilidad de incidir con sus comportamientos en las decisiones de las empresas y, por tanto, también en las posibilidades de difusión de la práctica de la responsabilidad social de empresa. Tal tendencia también se confirma en el caso de los consumidores italianos que, si bien en menor medida que los de otros países europeos, prefieren productos realizados en contextos productivos más respetuosos con el medio ambiente, con los derechos de los trabajadores y con las cuestiones sociales.

Según una investigación del Censis de 2003, los italianos tenían la siguiente idea de una empresa socialmente responsable: en la mayoría de los casos, el 36,2\%, "es una empresa que adopta sistemas productivos que no dañan el medio ambiente y la salud de los empleados y consumidores"; para el $31,2 \%$, en cambio, en una perspectiva más tradicional, la empresa responsable es aquella que "crea empleo, paga los impuestos y respeta las leyes "; para el 19,7\% la responsabilidad de la empresa debe volcarse en los empleados y por tanto "garantizar condiciones de trabajo óptimas a sus empleados, tutelando la salud, la promoción de iniciativas formativas y de crecimiento profesional y la oferta de servicios"; finalmente, para el $12,5 \%$ debe volcarse en la sociedad y por tanto promover iniciativas en el ámbito social de las que se beneficie la colectividad, con donaciones, favoreciendo la implicación de los empleados en iniciativas de voluntariado, organizando iniciativas culturales o de solidaridad. Según la misma investigación: a lo largo de 2002, casi un cuarto de las empresas italianas han apoyado activamente formas de voluntariado: cedien-

7 Cit. en Mario Molteni y Alessandra Todisco (al cuidado de): 'Piccole e Medie Imprese e CSR. La CSR come leva di differenziazione, Informe Altis - Isvi, 2007. 
do bienes $(25,7 \%)$ o favoreciendo la implicación del propio personal en actividades de este tipo (16,4\%); y además, el $45,8 \%$ ha patrocinado eventos culturales o deportivos, mientras que el $7,8 \%$ ha realizado campañas publicitarias o inversiones en la comunicación social ${ }^{8}$.

Pasando del plano general al sectorial, respecto a la adopción de la RSE en las empresas turísticas, la situación no es muy positiva, es decir, existe un retraso respecto a los demás sectores económicos.

Este retraso también ha sido señalado por el mundo de la investigación científica, que en los últimos años ha intensificado las investigaciones y los estudios sobre el turismo sostenible y responsable, del que se están desarrollando diversas iniciativas (convenios, acciones de formación y de actualización profesional de los operadores, etc.), también en colaboración con las asociaciones profesionales del turismo, orientadas al crecimiento de la cultura de la RSE.

Figura 1 - Los sectores del mercado más activos en la RSE en Italia.

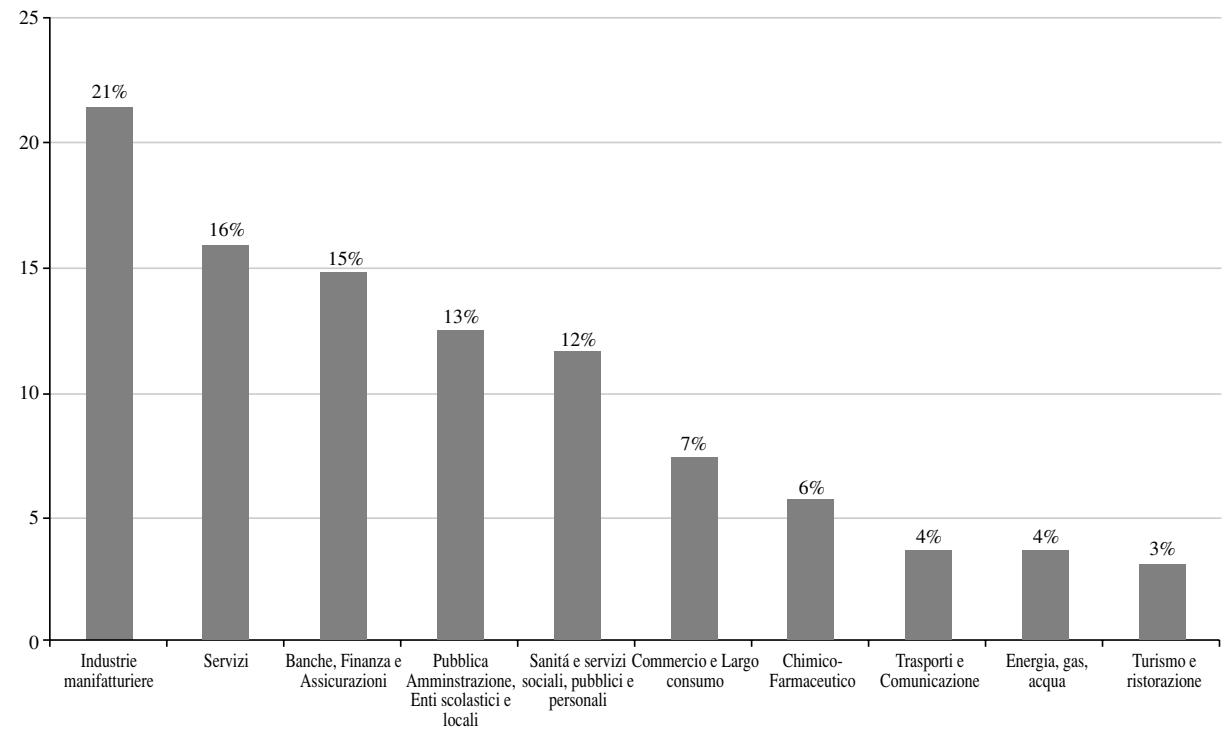

Leyenda:

- Sector manufacturero: incluye diversos sectores, entre los cuales: alimentos y bebidas; textil e indumentaria, aparatos mecánicos y eléctricos; automovilístico; papel, imprenta y editorial, construcción y cemento.

- Servicios: agencia de comunicación, inmobiliario, informático, servicios para el medioambiente.

- Sanidad y servicios públicos, sociales y personales: asociacionismo empresarial y distritos industriales, actividades recreativas, culturales y deportivas, fundación de empresa, sanidad y servicios sociales.

Fuente: Fundación Sodalitas, 2008

8 Cfr. Censis, Las empresas también producen capital social, 19 de junio de 2003, página www.censis.it 
A pesar de esto, el retraso todavía es elevado. El estudio realizado por la Fundación Sodalitas en 2008, señala los sectores del mercado más activos en la práctica de la RSE en Italia. El turismo está entre éstos pero, como se puede observar en la fig. 1, con el porcentaje de difusión más bajo $(3 \%)^{9}$.

Bajo el aspecto ético, la sensibilización también está pasando a través de acciones de carácter institucional. Por ejemplo, sobre el problema del llamado "turismo sexual", el Gobierno italiano en 2009 impulsará acciones de sensibilización, campañas audiovisuales y de implicación de los actores interesados, operadores del turismo en primer lugar, en una óptica de prevención. A la campaña, que recoge los compromisos asumidos en el Tercer Congreso Mundial contra la explotación sexual de los niños y adolescentes, celebrado en Río de Janeiro en 2008, participó Ecpat Italia Onlus (End Child Prostitution Pornography and Trafficking), una de las principales asociaciones en el mundo sobre este tema.

Pero el factor que mayormente está contribuyendo a la sensibilización de los empresarios turísticos italianos (hoteleros, hosteleros, tour operadores, agencias de viaje, etc.) sobre la RSE, no es tanto de orígen ético, sino de mercado. Cuestiones como la contaminación de los mares, la degradación paisajística, la excesiva carga antrópica, la banalización de las relaciones host/guest, la excesiva estandarización de los productos turísticos, etc., son los temas en los que se ha centrado la atención del turista/consumidor, que tiende a preferir las localidades turísticas en las que ese tipo de problemas estén menos acentuados. Los empresarios del turismo se están dando cuenta de que las posibilidades de éxito de su empresa cada vez están más relacionadas con la capacidad de adecuarse a los principios de la RSE, desde un punto de vista concreto, y no como operación fachada (de imagen).

Entre las grandes empresas turísticas italianas un tour operador ha seguido el camino de la sostenibilidad, "I Viaggi del Ventaglio" (Los viajes del abanico) líder en el mercado italiano y cotizada en Bolsa, que se ha dotado de una "Sección turismo y medio ambiente" que tiene como objetivo coordinar las iniciativas orientadas a promover un cambio cultural en la estructura de la empresa, la sensibilización de los partners, de las agencias de viajes, de los hoteleros y de los clientes. La experiencia más significativa es el laboratorio empresarial para el turismo sostenible, una experiencia piloto en la que participa la estructura turística "Playa Maroma" situada en las cercanías de Cancún, en la Riviera Maya mexicana (en un área de gran valor natural y paisajístico, amenazada por el reciente e intensivo desarrollo turístico). Una experiencia sobre cuestiones relacionadas con la sostenibilidad (en particular con el respeto del medio ambiente), que ha

9 El estudio "La CSR in Italia. Alcune tendenze in atto" (La RSE en Italia. Algunas tendencias actuales) ha sido realizado por la Fundación Sodalitas en octubre de 2008. Ha tenido como objetivo "recoger datos y describir el contexto en el cual se desarrolla la RSE, obteniendo información, tanto sobre las empresas más sensibles a este tema (dimensión, sector de pertenencia, posición geográfica), como sobre proyectos realizados por éstas, poniendo en evidencia los ámbitos de intervención mayormente elegidos”. El análisis se ha realizado en base a una muestra de 541 empresas y 972 proyectos. En la mayor parte de los casos se trata de empresas de grandes dimensiones (64\%). Las Pequeñas y Medianas Empresas (PMI) y la Administración Pública (PA) completan la muestra con el $19 \%$ y el $17 \%$ respectivamente. 
implicado a muchos stakeholders locales y no locales como sujetos activos (proyectistas, biólogos, partners comerciales, empresas constructoras, etc.).

Para los objetivos de este trabajo, teniendo en cuenta la estructura típica del sistema empresarial italiano, se ha decidido concentrar la atención en las empresas turísticas pertenecientes a la categoría de las PMIs. Como consecuencia de la limitada difusión de la RSE en el sector turístico citada anteriormente, no ha sido fácil encontrar un caso de estudio particularmente significativo. El análisis documental, en particular de la documentación de las sociedades especializadas en la RSE en Italia, el informe publicado más importante y, sobre todo, la mayor visibilidad (vía internet) de la RSE de las empresas turísticas que la practican, nos han llevado a elegir el "Consorzio Piccoli Alberghatori di Rimini" (Consorcio de Pequeños Hoteleros de Rímini) que, además, es una agrupación de PMIs. Este es el caso de estudio que se analizará a continuación.

\section{ESTUDIO DE UN CASO: EL CONSORCIO 'PICCOLI ALBERGHI DI QUALITÀ DI RIMINI'10}

\subsection{PREMISAS}

En su Libro Verde, la Comisión Europea, no sólo se preocupa por dar una definición de la RSE, sino también por establecer campos de aplicación, ámbitos de intervención y objetivos: se invita a las empresas a responder directamente a las exigencias de una sociedad en continua evolución y más interesada, no sólo en las temáticas medio ambientales, sino también en las relaciones sociales/humanas.

El análisis de este consorcio, ha permitido evidenciar aspectos relacionados con la RSE en Italia, algunos de los cuales es oportuno recordar antes de seguir adelante:

a) es posible detectar casos en los que, a través de mecanismos internos, no reglamentados y espontáneos, el empresario - con una sensibilidad innata hacia esta temática - decide anteponer "la ética y la responsabilidad", a las prácticas empresariales. Sin embargo, normalmente no es así. Entonces, la adopción de los principios de la RSE sólo es posible a través de la creación de reglas formales de autorregulación (como códigos éticos, balances sociales, etc.);

b) para que todo esto no sea mera retórica, es necesaria la comunicación hacia el exterior de tal compromiso, principalmente por dos razones: para permitir que los diferentes stakeholders interesados en tales temáticas conozcan la existencia de la empresa misma (condición necesaria) y el tipo de compromiso asumido; porque proporcionar información sobre los valores de la empresa, los servicios y los productos que ofrece, es un ejemplo positivo para otras empresas.

En referencia al contexto italiano, la situación de la RSE impide la realización de una valoración clara. Lo que sin duda parece evidente, es el gran aumento del

10 Este apartado lo ha escrito Elena Giglio, Licenciada en "Ciencias Turísticas" y estudiante de "Valorización de los Sistemas Turístico-Culturales". Colabora con el Departamento de Sociología y de Ciencias Políticas Centro Investigaciones y Estudios sobre el Turismo, Universidad de Calabria, E-mail: egiglio@live.it. 
interés sobre el tema, en especial en los últimos años. De la misma manera que el aumento de la visibilidad de prácticas de RSE depende más de la utilidad instrumental que se obtiene, que no de la efectiva convicción de la utilidad social de la misma.

c) La carencia de comunicación y visibilidad se puede explicar de dos formas:

1 - No difundir las actividades de RSE depende de que las empresas no quieren ser "percibidas" como empresas que utilizan estrategias cínicas ${ }^{11}$, ya que son víctimas de miedos sociales (óptica confiada).

2 - No difundir las actividades de RSE, depende del hecho que las empresas han adoptado políticas de RSE sólo por razones de imagen, y no porque estén convencidas de la función de utilidad social implícita en las prácticas de RSE (óptica desconfiada).

Probablemente, como algunos señalan, la respuesta justa es una vía intermedia entre el pudor de quien adopta prácticas de RSE y no entiende la finalidad y los resultados, y entre el cinismo de quien las adopta pensando sólo en la posibilidad de aumentar los beneficios de la empresa.

Estas consideraciones y valoraciones se refieren a las empresas italianas en su conjunto y, por tanto, también a las del sector turístico. Nuestra impresión es que en el turismo, aunque también existan empresas orientadas hacia la RSE, el fenómeno de la falta de comunicación citado anteriormente, es todavía más acentuado. Esta conclusión se basa en la realización de un minucioso análisis en la web: ha resultado dificilísimo encontrar empresas turísticas relacionadas con las palabras clave: balance social, código ético, RSE.

Ya que el objetivo de este apartado es la descripción de un caso de empresa turística comprometida con la RSE, hemos decidido concentrarnos en el estudio de un caso que en nuestra opinión, puede proporcionar información importante sobre el significado de la RSE en la empresa turística italiana, en particular de las PMIs. En base a estas consideraciones hemos seleccionado el "Consorzio Piccoli Alberghi di Qualità di Rimini”.

\subsection{ORGANIZACIÓN, ÁMBITOS DE INTERVENCIÓN, INNOVACIÓN.}

Emilia Romaña es una de las regiones italianas con mayor experiencia en el campo de la RSE en el turismo, tanto por la presencia de un know-how empresarial óptimo, como por la importante presencia del turismo en la misma.

El territorio siempre ha sabido renovar la propuesta vacacional siguiendo los cambios de un sector, como lo es el sector turístico, basado en exigencias y deseos en continuo movimiento. La atención hacia estos cambios ha estimulado el desarrollo de experiencias de asociacionismo y experimentos económico-comerciales interesantes, como el

11 Comisión Europea, Dirección General, Guía para una comunicación eficaz.

12 Los Clubes de Producto son organizaciones de carácter regional o nacional formadas por una pluralidad de empresas que se unen para perseguir y alcanzar objetivos comunes, como aumentar la propia visibilidad en el mercado, incrementar sus ganancias, conquistar nuevos nichos de mercado internacional. 
desarrollo del Club del Producto ${ }^{12}$, y también actividades innovadoras relacionadas con el medio ambiente (por ejemplo, el municipio de Rímini cuenta con un depurador desde los años 70).

En este contexto, es particularmente interesante el caso del "Consorzio Piccoli Alberghi di Qualità di Rimini”, creado en 1999 en Rímini, con el objetivo de valorizar y promover las pequeñas estructuras de la costa Adriático-Romañola, o sea: Bellaria, Igea Marina, Rimini, Riccione, Cattolica y Misano.

El Consorcio, formado por 65 hoteles, prevé como criterio de selección el tamaño (máx. 48 habitaciones), además de compartir, firmar y respetar el reglamento-disciplinar del propio Consorcio. El reglamento prevé una serie de compromisos, diversificados en base a la clasificación:

- atención hacia la persona, a través del suministro de servicios personalizados;

- atención hacia el territorio, a través del compromiso de utilización de algunos productos típicos locales con el objetivo de valorizar las producciones locales, proponiendo al menos una vez a la semana cenas o comidas a base de platos tradicionales. Se ha llevado a cabo una recogida de recetas tradicionales (más de cien), que se ha repartido en los hoteles del consorcio;

- atención hacia el medio ambiente, a través de la estimulación del ahorro energético, la recogida selectiva de residuos, el uso de productos locales;

- verificación y valoración del respeto del Reglamento a través de visitas y controles periódicos.

Fig. 2 - Área geográfica de intervención del "Consorzio Piccoli Alberghi di Qualità"

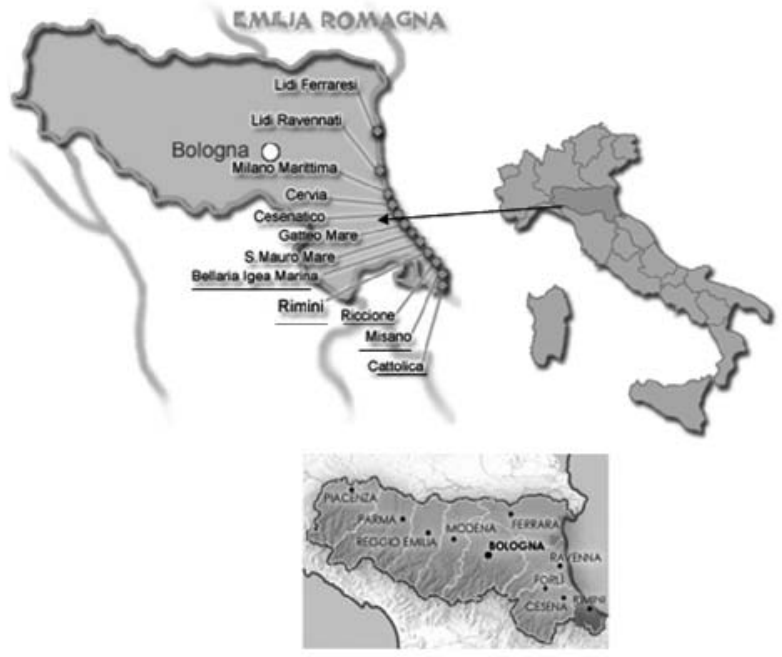

Fuente: elaboración propia con Google maps 
Después de autorreglamentarse en la fase inicial a través de un "código interno", en julio de 2002 el Consorcio ha obtenido el certificado ISO 9001:2000", que ha renovado en 2005 para las siguientes actividades:

- apoyo organizativo a los socios para la proyectación y la oferta de actividades;

- control y verificación de los servicios suministrados a todos los hoteles asociados.

Marta Agostini, Presidenta del Consorcio, dice que: “...la misión del Consorcio es poner en el centro de todo a la "persona", reconocida no sólo en el cliente, sino en general, en todos los stakeholders, teniendo como objetivo el desarrollo de relaciones transparentes respecto a todos los actores y la atención hacia el medio ambiente y las comunidades locales. De ésto deriva el reconocimiento de los tres valores de referencia: clientes, territorio, instituciones".

\subsection{AMBIENTE INTERNO Y EXTERNO, RELACIONES CON LA COMUNIDAD.}

La definición de RSE deja espacio a las empresas para elegir autónomamente las áreas de intervención en las que actuar. Tal y como aparece en el Libro Verde, algunos de los objetivos que un empresario éticamente responsable debe respetar son: el empleo razonable de los recursos humanos; el respeto de los derechos de los trabajadores; la ausencia de formas de discriminación sexual, religiosa y racial; la tutela de la salud; la promoción de los recursos humanos y del capital intelectual y humano interno a la empresa. Si se adoptan tales opciones, éstas ponen en marcha un proceso virtuoso que promete una ventaja competitiva para quien las aplica, por ejemplo: una mayor satisfacción en los negocios; una facilidad mayor a la hora de reclutar nuevos talentos; un aumento de la fidelidad de los empleados; mayor motivación y por lo tanto, mayor compromiso; productividad; eficiencia y colaboración. Los tres ámbitos de intervención del Consorcio respecto a la RSE son: el ambiente interno, el externo y las relaciones con la comunidad.

El Consorcio, a través de encuentros dirigidos al intercambio de experiencias, problemáticas y soluciones, y a través de cursos de profundización orientados hacia los asociados sobre temáticas señaladas por ellos mismos, ha conseguido crear un clima de colaboración y cordialidad entre los socios, que favorece el crecimiento de las empresas y contribuye al desarrollo del Consorcio mismo. Son un ejemplo los seminarios realizados en colaboración con la Cámara de Comercio de Rímini ${ }^{14}$ y con la Asociación Figli del Mondo ${ }^{15}$ llamados "Incontrarsi", en los cuales se han ilustrado las experiencias llevadas a cabo en el sector de la Responsabilidad Social en el territorio de Rimini.

13 Recogida en las normas ISO 9000 que reconoce el "léxico" para la 9001 y la 9004. La ISO 9001 se ocupa de la definición de los requisitos de los sistemas de calidad y prevé una orientación global y completa del certificado, por lo que no es posible excluir sectores o procesos empresariales de la organización o necesarios para satisfacer a los clientes. La ISO 9004 es una línea guía para la mejora de las prestaciones de las organizaciones. 14 Cámara de Comercio de Rimini, Ente público autónomo que tutela los intereses generales de las empresas comerciales y promueve el desarrollo económico del territorio provincial.

15 La asociación de Promoción Social "Figli del Mondo" se fundó en abril de 2002 como iniciativa sin ánimo de lucro promovida por un grupo de empresarios, dirigentes de empresa y profesionales, operantes en el terri- 
Con referencia al ambiente natural, la RSE tiene como objetivo primario la sensibilización hacia las temáticas medio ambientales y el desarrollo en clave sostenible de múltiples actividades. En tal ámbito, la acción del Consorcio se ha dirigido hacia actividades que tienden a utilizar la gran cantidad de recursos del territorio y, sobre todo, su buena gestión a lo largo del tiempo. Un ejemplo es el reconocimiento de la llamada "Bandiera Blu"(Bandera Azul) ${ }^{16}$, que se concede a las localidades costeras con una alta calidad de sus aguas, y que desde hace algunos años se asigna a las localidades en las que se ubican las estructuras hoteleras del Consorcio ${ }^{17}$.

El compromiso de los hoteleros se basa en un conjunto de buenas prácticas, acerca de las cuales los socios están sensibilizados y formados:

1. Basura. Compromiso para la reducción de la cantidad de residuos producidos a través de la adquisición de: productos poco empaquetados; rellenado de detergentes y otros productos líquidos; productos de proveedores que quieren dotarse del certificado Ecolabel; participación en la recogida selectiva de los residuos.

2. Agua. Compromiso en la adopción de medidas de ahorro hídrico, a través de la instalación de aceleradores de flujo para las duchas, grifos y válvulas de distribución que permiten un mejor servicio y un sustancial ahorro hídrico y energético (calentar el agua). Compromiso en la promoción de comportamientos idóneos entre la clientela.

3. Energía. Promoción del ahorro y la recuperación energética a través de la reducción de la ropa destinada al lavado. Progresiva instalación de bombillas de ahorro energético.

4. Alimentación. Compromiso a favorecer el uso de alimentos sin sustancias químicas (pesticidas y anticriptogámico). Compromiso a no utilizar alimentos genéticamente modificados. Sensibilización hacia el uso de productos del circuito Equo solidario.

5. Gastronomía. Valorización de las especialidades gastronómicas del territorio mediante la promoción y la oferta de platos regionales preparados con productos típicos.

torio de la provincia de Rimini. Figli del Mondo se dirige a las Asociaciones empresariales, a las empresas, a entes e instituciones, a profesionales y a particulares que reconozcan el significado y el valor de una forma diferente de hacer empresa, y cuyas capacidades organizativas, proyectuales y económicas, se empleen a favor de toda la comunidad.

16 La Bandera Azul es un reconocimiento concedido por la FEE (Foundation for Environmental Education) a las localidades costeras europeas que satisfagan algunos parámetros establecidos en base a criterios de calidad en las zonas de baño, sin olvidar la limpieza de las playas y de los puertos turísticos. Instituida en 1987, año europeo del medio ambiente, la campaña Bandera Azul la llevan a cabo, en todos los estados europeos, los órganos locales de la FEE que, a través de un Comité nacional, efectúan visitas de control en las ciudades candidatas, para después proponer a la FEE Internacional las candidaturas.

17 Rimini, Riccione y Misano Adriatico han obtenido la Bandera Azul durante seis años consecutivos; Bellaria e Igea Marina durante tres años consecutivos; Cattolica durante 12 años consecutivos. 
6. Transportes. Promoción del uso de medios de transporte colectivo público y privado, incluso con la difusión de billetes específicos puestos a disposición por las empresas de transporte, y la disponibilidad de bicicletas para los huéspedes.

7. Desplazamientos. Promoción del uso de pistas y caminos ciclo-peatonales. Alquiler y/o cesión gratuita de bicicletas.

8. Ruidos. Compromiso a reducir los ruidos producidos en el interior del establecimiento y en las áreas pertinentes, sobre todo en horario nocturno, incluso mediante una acción de promoción de comportamientos idóneos para con los clientes/turistas. Compromiso a sostener eventuales iniciativas públicas dirigidas a disminuir el ruido en las áreas adyacentes al establecimiento.

9. Bienes Culturales. Compromiso a participar en la valorización de las propuestas turísticas alternativas y en la promoción de los bienes culturales (itinerarios naturales y paisajísticos, visitas a monumentos y/o lugares importantes del territorio a nivel histórico).

Además, algunos hoteles del Consorcio también adoptan el reglamento disciplinario de Legambiente ${ }^{18}$. En este caso se identifican con el logo de Legambiente, tanto en el interior del hotel como en la página web del Consorcio.

Con referencia a las relaciones con la comunidad local, el compromiso RSE puede desarrollarse de diferentes maneras: a través de la elección de proveedores locales; a través del control de toda la cadena productiva y en consecuencia la adquisición de productos socialmente responsables; a través del apoyo a organizaciones sin ánimo de lucro (Onlus) o de voluntariado, locales o nacionales.

En este campo, el Consorzio Piccoli Alberghi di Qualità, desde hace algunos años, organiza veladas de solidaridad para obtener fondos destinados a ayudar a las Onlus que operan en el territorio riminense. A lo largo de los años han llevado a cabo las siguientes iniciativas:

- RiminiAil: junto con la "Associazione Italiana contro Leucemie, Linfoma e Mieloma" (Asociación Italiana contra Leucemia, Linfoma y Mieloma), para recoger fondos y así poder proporcionar un médico especializado que se desplace al domicilio de los enfermos y efectúe transfusiones en los casos más graves.

- Crescere Insieme: en colaboración con "Associazione genitori di persone con sindrome di Down" (Asociación de padres de personas con síndrome de Down), para la recogida de fondos destinados a encontrar profesionales a incluir en proyectos específicos (por ejemplo: "Percorsi abilitativi" (Itinerarios de habilitación), dirigido a chicos/as hasta los 18 años; "Indipendentemente" dirigido a chicos/as adolescentes y adultos).

18 Legambiente es una asociación ambientalista italiana nacida en 1980 en el ámbito del ARCI (Associazione Ricreativa e Culturale Italiana, asociación de promoción social), y sucesivamente se ha hecho autónoma. Con más de 115.000 socios y 1.000 círculos locales es la asociación ambientalista italiana más importante, está reconocida por el Ministerio del Medio Ambiente y forma parte de la Oficina Europea del Medio Ambiente y del International Union for Conservation of Nature. 
- A.R.O.P.: en colaboración con la "Associazione Riminese Oncoematologia Pediatrica" (Asociación Riminense de Oncohematología Pediátrica) y otras enfermedades crónicas de la infancia, para sostener la investigación científica.

- Figli del Mondo: en colaboración con la "Associazione Figli del Mondo" (Asociación Hijos del Mundo), organización orientada a la creación de relaciones entre empresas con ánimo de lucro y asociaciones sin ánimo de lucro. El Consorcio ha llevado a cabo iniciativas para ayudar a madres jóvenes y a menores en riesgo (Casa de acogida para ayudar a las dos categorías poco favorecidas; bonos de compra para la adquisición del ajuar básico del recién nacido o de juguetes para el niño, etc.).

- I.O.R.: en colaboración con el "Istituto Oncologico Romagnolo"(Instituto Oncológico Romañolo) se encarga de investigar y dar asistencia a los enfermos oncológicos. En las Navidades de 2008, el Consorcio ha donado una gran cantidad de copias del libro "Piccole ricette di Qualità", de cuya venta se han recabado fondos destinados a la investigación.

- ISAL: en colaboración con la "Associazione a sostegno della cura e studio del dolore" (Asociación para el tratamiento y el estudio del dolor), el Consorcio ha organizado una recogida de fondos destinados a la investigación y a la sección Hospice (asilo) del hospital de Rímini.

\subsection{EL CAMBIO DE LA EMPRESA: EL CASO DEL HOTEL EDY}

Ya hemos indicado que uno de los objetivos del Consorcio es sensibilizar y estimular a los asociados para desarrollar prácticas relacionadas con la RSE. Con este propósito, la experiencia de uno de los afiliados, el Hotel Edy, es emblemática,.

El hotel ha recibido recientemente reconocimientos significativos: ha sido citado en un artículo de la conocida y acreditada guía gastronómica "Gambero Rosso"19, por su atención hacia el cliente y por el cuidado y la calidad de su cocina; ha sido finalista del premio Sodalitas ${ }^{20}$. Estos reconocimientos se le han otorgado por el clima familiar y hospitalario de la estructura.

El Hotel Edy está comprometido con acciones de sensibilización y orientación hacia la problemática medio ambiental, que han llevado: al ahorro hídrico y energético (reducción de la cantidad de basura producida; utilización de productos poco empaquetados; utilización de detergentes ecocompatibles, como las microesferas de cerámica; preferencia de productos con certificación Ecolabel; instalación de nuevas tecnologías,

19 Conocida revista gastronómica italiana nacida en 1986. Cfr. el número de julio de 2002.

20 El Premio, reconocido por el Presidente de la República italiana, se dirige a las empresas, asociaciones de empresarios, distritos indutriales, etc, concretamente comprometidos en la cuestión social a través del desarrollo de comportamientos éticos y de la aplicación de valores declarados. Se divide en 7 categorías (ej.: Premio para el mejor programa de responsabilidad social orientado a la valorización del capital humano; Premio para la mejor iniciativa de sostenibilidad; Premio para la realización del mejor programa de partnership en la comunidad, etc.). Todos los proyectos candidatos se publican en "Libro de Oro de la Responsabilidad Social" - el volumen que recoge las best practices de RSE - y en la página web www.sodalitas.socialsolution.it. 
como aceleradores de flujo para duchas, grifos y válvulas de distribución en general; disminución de la ropa destinada al lavado; montaje de bombillas de ahorro energético; participación activa en programas de reciclaje, etc.); a una mayor atención y colaboración por parte del personal interno en el uso correcto de detergentes, en el uso atento del agua $\mathrm{y}$, sobre todo, en la correcta recogida selectiva de los residuos (orgánicos, vidrio, plásticos, papel, etc.); al aumento de la calidad del servicio ofrecido y de los estándares garantizados, en la óptica de la sostenibilidad.

Respecto a la clientela, los gestores no sólo promueven el uso de medios de transporte públicos, sino que también ponen a su disposición medios de transporte no contaminantes, como las bicicletas. Respecto a la comunidad local, el Hotel está comprometido principalmente en dos frentes: el respeto de las tradiciones y del territorio y el compromiso con lo social.

De hecho, el hotel se caracteriza no sólo por la calidad de sus menús, sino también por la atención que presta a la cultura gastronómica del territorio. Una demostración práctica es la organización de veladas temáticas, en las que cada plato tradicional servido se acompaña por la narración de sus orígenes. Además, pone a disposición de sus clientes productos eno-gastronomicos típicos del territorio, como el aceite extra virgen de oliva obtenido mediante el prensado en frío, vinos DOC $^{21}$ producidos por empresas agro turísticas de las colinas del interior de la región, alimentos biológicos de productores locales. Valoriza propuestas turísticas alternativas como la "Strada dei Vini e dei Sapori" 22 (Ruta de los Vinos y de los Sabores), y rutas turístico/monumentales que pasan por los lugares de la herencia histórica romañola.

En cuanto al compromiso social, el Hotel Edy, junto con otros hoteles del Consorcio, participa en la fiesta anual de los socios "Coriandoli di Solidarietà" (Confeti de Solidaridad), en la que se recogen fondos destinados a proyectos locales, normalmente de carácter socio-sanitario.

El Hotel Edy ha contribuido, en 2004, junto con todos los hoteles asociados del Consorcio, a la obtención del premio "Adriatic Coast Award"23 en la sección: "La agrupación de empresas de la Riviera que mejor representa los conceptos del Club: cooperación, innovación, identidad común y homogeneidad de la oferta" con la siguiente moti-

21 Acrónimo de "Denominación de Origen Controlada". Es una marca de origen que certifica la zona de origen delimitada de la recogida de las uvas utilizadas para la producción del vino; se utiliza para designar un producto de calidad y renombre, cuyas características están relacionadas con el ambiente natural y los factores humanos. La categoría de los vinos DOC incluye los vinos producidos en determinadas zonas geográficas respetando una forma específica de producción (aprobada por Decreto Ministerial). Tales vinos, antes de ser comercializados, deben ser sometidos, en la fase de producción, a un análisis químico-físico preliminar y a un examen organoléptico que certifique que se respetan los requisitos previstos por el estándar reglamentario; el incumplimiento de los requisitos impide la comercialización con la sigla DOC.

22 Itinerarios efectuados en colaboración con entes, instituciones, asociaciones de categoría y empresas privadas, que tienen el objetivo de valorizar la identidad histórica, cultural, ambiental, económica y social del territorio de pertenencia.

23 Reconocimiento anual promovido por la Unión de Prodotto Costa (Producto Costa, asociación formada por municipios, provincias y operadores turísticos de estos territorios), para premiar a los operadores por la hospitalidad de la Riviera Romañola. 
vación: "han sido capaces de valorizar la oferta típica y familiar de la Riviera romañola que, hasta hace pocos años, se consideraba marginal, dando, en cambio, valor propio a esta característica, y también aplicando sistemas de calidad y llevando a cabo proyectos innovadores". El Hotel ha sido elegido por la Cámara de Comercio de Rímini para participar en la edición 2008 de Sodalitas Social Solution ${ }^{24}$, proyecto de la Fundación Sodalitas $^{25}$, realizado en colaboración con Impronta Etica ${ }^{26}$.

\subsection{CONSIDERACIONES FINALES}

El análisis realizado, evidencia una situación que no deja lugar a dudas: en el caso del "Consorzio Piccoli Alberghi di Qualità di Rimini”, la aplicación de los principios de la responsabilidad social de empresa es algo concreto, y no sólo una operación instrumental y de imagen.

$\mathrm{Su}$ forma de operar y la de cada uno de sus afiliados se basa en la dirección indicada en el Libro Verde de la Comisión Europea, ya que ha estimulado el nacimiento de una nueva cultura y, por tanto, a su vez, de nuevas maneras de comportarse y considerar: el medio ambiente, la misión de la empresa, las comunidades locales, la vida social, el territorio.

Aunque todavía queda mucho por hacer para alcanzar una vasta difusión en todos los sectores empresariales de esa cultura de RSE necesaria en un mundo que ve en las palabras globalización y sostenibilidad el presente y el futuro de la sociedad, ejemplos como el del Consorcio pueden, o más bien deben, representar ejemplos positivos a imitar, sobre todo en el sector turístico que, como en el caso del ejemplo italiano, puede contar con un territorio rico en potencialidades y en recursos artísticos, naturales y monumentales.

El caso del "Consorzio Piccoli Alberghi di Qualità di Rimini”, nos parece que confirma la posibilidad de demostrar una concreta atención respecto a las preocupaciones sociales de las personas manteniendo, al mismo tiempo, inalteradas, las propias oportunidades de negocio y, por tanto, mejorando decididamente la propia reputación a los ojos de todos los portadores de intereses.

\section{BIBLIOGRAFÍA}

Bagnoli, L. (2004): Quale responsabilità d'impresa?, Milán, Franco Angeli. Bauman, Z. (1999): La società dell'incertezza, Boloña, Il Mulino.

24 Promovido por la Fundación Sodalitas, premia proyectos excelentes de Corporate Social Responsibility realizados en el mercado italiano.

25 Sodalitas nace en 1995, gracias a la iniciativa de Assolombarda, de un grupo de empresas y de algunos directores, para crear un nexo entre el mundo de la empresa y el no-profit. En 2008 se convierte en Fundación. 26 Impronta Etica nace en Bolonia en 2001, como asociación sin ánimo de lucro comprometida con la promoción y el desarrollo de la Responsabilidad Social de Empresa. Impronta Etica crea una red entre empresas y organizaciones que consideran el compromiso social un elemento esencial de la propia actividad y aplican prácticas de Responsabilidad Social. 
Bauman, Z. (2001): Voglia di comunità, Bari, Laterza.

Beda, A. Bodo, R. (2004): La responsabilità sociale d'impresa. Strumenti e strategie per uno sviluppo sostenibile dell'economia, Milán, Ed. Il Sole 24 ore.

Brecher, J., Costello, T. (2001): Contro il capitale globale, Milán, Feltrinelli.

Becker, U. (2000): I rischi della libertà, Boloña, Il Mulino.

Burlingame, D. (2002): “Corporate giving”, in International Journal of Nonprofit and Voluntary Sector Marketing, vol. 6, n. 1: 4-5.

Capecchi, V. (2005): La responsabilità sociale dell'impresa, Roma, Carocci.

Carrol, A. B. (1991): "The pyramid of Corporate Social Responsibility", Business Horizons, n. 34.

Cattaneo, C. (al cuidado de) (2003): Il Bilancio Sociale nell'evoluzione dei rapporti tra economia e società, Milán, Giuffrè Editore.

Cerana, N. (al cuidado de) (2004): Comunicare la responsabilità sociale. Teorie,modelli, strumenti e casi d'eccellenza, Milano, Franco Angeli.

Chiesi, A., Martinelli, A., Pellegatta, M. (2000): Il bilancio sociale. Stakeholder e responsabilità sociale d'impresa, Milán, Ed. Il Sole 24 ore.

Cisi, M. (2000): Il Bilancio ambientale, Turín, Giappichelli Editore.

Commissione Comunità Europea (2001): Green paper. Promoting a Eeuropean Framework for Corporate Social Responsibility, Bruselas, Comisión Europea.

D’Orazio, E. (al cuidado de) (2003): La responsabilità sociale d'impresa: teorie,strumenti, casi, Fascículo especial de "Notizie di Politeia", vol. 72.

Freeman, R. E. (1984), Strategic management: a stakeholder approach, Boston, Pitman.

Frey, M. (a cura di) (2004): La responsabilità sociale d'impresa nella prospettiva dei consumatori, Milano, CCIAA de Milán.

Gallino, L. (2007): Prospettive della Responsabilità sociale delle imprese. Il contesto internazionale, verso una nuova definizione di Responsabilità Sociale, Roma, Fundación I-RSE.

Geertz, C. (1995): Mondo globale, mondi locali, Boloña, Il Mulino.

Invernizzi, E. (2005): Manuale di relazioni pubbliche - Le tecniche e i servizi dibase, Milán, McGraw-Hill.

Kotler, P., Roberto E. L. (1991): Marketing sociale. Strategie per modificare I comportamenti collettivi, Milán, Edizioni di Comunità.

Lodolo D’Oria, V. (a cura di) (2001): La sponsorizzazione: dagli enti locali alla sanità. Il volto nobile del marketing, Milán, Franco Angeli.

Manfredi, F. (1997): Le sponsorizzazioni sociali, Milán, Etas.

Marzantonio, R., Tagliente, F., (2003): Il bilancio sociale dell'impresa responsabile.Scelte economiche nel rispetto dell'uomo e dell'ambiente, ............. , Maggioli.

Michelini L. (2003): Strategie di corporate giving e cause related marketing in Italia: tra benessere sociale e fini di business, Actas del Congreso Internacional "Le tendenze del marketing", Venecia. 
Ministero del Lavoro e delle Politiche Sociali (2003): Progetto RSE-Sc: il contributo italiano alla campagna di diffusione della RSE in Europa, Tercera conferencia Europea sobre la RSE, Venecia.

Molteni, M. (al cuidado de) (2004): Secondo Rapporto sulla Responsabilità Sociale d'Impresa in Italia, Milán, ISVI - ALTIS.

Molteni, M., Devigili, D. (2004): Il cause related marketing nella strategia d'impresa, Milán, Franco Angeli.

Molteni M., Lucchini M. (2004): I modelli di responsabilità sociale nelle imprese italiane, Ricerca Unioncamere-ISVI, Milán, Franco Angeli.

Muzi Falconi, T. (2002): Governare le relazioni, Milán, Ed. Il Sole 24 Ore.

Pittèri, D., Picucci, S., Villani, R.M. (2002): Cause Related Marketing. Agire competitivo dell'impresa e nuovo mercato, Milán, Franco Angeli.

Rusconi G. (1988): Il bilancio sociale d'impresa, Milán, Giuffrè Editore.

Sassen, S. (2002): Globalizzati e scontenti, Milán, Il Saggiatore.

Sennet, R. (2001): L'uomo flessibile, Milán, Feltrinelli.

Shiva, V. (2004): Le guerre dell'acqua, Milán, Feltrinelli.

Unioncamere - Centro Studi (2006): La responsabilità sociale delle imprese e gli orientamenti dei consumatori, Milán, Franco Angeli.

Viviani, M. (1999): Specchio magico. Il bilancio sociale e l'evoluzione delle imprese, Boloña, Il Mulino.

\section{PÁGINAS DE INTERNET CONSULTADAS}

www.bilanciosociale.it

www.clubsocialis.org

www.cseurope.org

http://www.edyhotel.it/index.html

www.gruppobilanciosociale.org

www.ilsole24ore.com

www.improntaetica.org

www.isvi.org

www.itconsult.it

www.italiaoggi.it

www.orsadata.it

www.piccolialberghi.com

http://www.riminieconomia.it/cciaa

www.sa8000.info

www.socialpress.it

www.sodalitas.it

www.welfare.gov.it

http://whc.unesco.org 